\title{
Phase measurements with wide-aperture interferometers
}

\author{
Arnaud Dubois, Juliette Selb, Laurent Vabre, and Albert-Claude Boccara
}

\begin{abstract}
An interferogram produced by wide-aperture interferometers is studied both theoretically and experimentally. The fringe spacing is shown to increase nonlinearly with the numerical aperture and the fringe envelope to become narrower as the numerical aperture is increased. Phase measurements with wide-aperture interferometers therefore require calibration, and the phase can be measured only over a limited range. A calibration is given for accurate phase measurements, and the range over which the phase can be measured is calculated. Experimental measurements are presented and compared with theory. (C) 2000 Optical Society of America
\end{abstract}

OCIS codes: $120.5050,120.2830,120.3180,180.3170$.

\section{Introduction}

Phase measurements are extremely useful for testing high-precision optical systems. A wide range of physical parameters such as stress, vibration, displacement, height, and surface profile can be obtained. Because of the short wavelength of optical radiation $(\lambda \sim 0.1-10 \mu \mathrm{m})$, the measurement of the optical phase provides information that is among the most precise possible. For example, height measurements with $\sim 1-\AA$ resolution can be achieved. ${ }^{1}$ Moreover, phase measurements are noncontact methods, capable of providing real-time nondestructive and whole-field information. Phase measurements have been performed with almost all types of interferometer systems. The main difficulty is accurate extraction of the phase from the measurement of the interference signal. For that purpose many methods have been developed. ${ }^{2}$ For all methods a discrete or continuous temporal phase shift is introduced. By measuring the intensity of the interference signal as the phase is shifted, we can obtain the phase. All methods assume knowledge of the relationship between the intensity of the interference signal and its phase. In the usual case, assuming monochromatic plane waves, the interference signal

The authors are with Ecole Supérieure de Physique et Chimie Industrielles, Laboratoire d'Optique, Centre National de la Recherche Scientifique, Unité Propre de Recherche A0005, 10 rue Vauquelin, F-75231 Paris cedex 5, France. A. Dubois's e-mail address is dubois@optique.espci.fr.

Received 12 July 1999; revised manuscript received 21 January 2000.

0003-6935/00/142326-06\$15.00/0

(C) 2000 Optical Society of America intensity varies sinusoidally with the phase. However, in wide-aperture interferometers such as Michelson, Mirau, or Linnik interference microscopes shown schematically in Fig. 1, the interference signal intensity no longer varies sinusoidally with the phase. The fringe spacing increases with the numerical aperture, and the fringe contrast vanishes with the optical path difference more and more rapidly as the numerical aperture is increased. ${ }^{3-9}$ Therefore corrections should be used with the usual methods to get the phase accurately. In addition, the phase cannot be measured over an illimited range.

We present a theoretical analysis of the optical intensity response of wide-aperture interferometers. Corrective factors for accurate phase measurements are given. The range over which the phase can be measured is calculated. Experimental results are presented and compared with theory.

\section{Theory}

In double-pass interferometers in which the illumination beam makes with the object and the reference surfaces an angle of incidence $\theta$, the interference signal intensity $I(z)$ as a function of the distance $z$ between the two surfaces is

$$
I(z)=A_{O}{ }^{2}+A_{R}{ }^{2}+2 A_{O} A_{R} \cos (2 k z \cos \theta+\phi),
$$

where $A_{O} \exp \left(i \phi_{O}\right)$ and $A_{R} \exp \left(i \phi_{R}\right)$ are the complex amplitudes of the waves after reflection on the object and the reference surfaces, respectively; $\phi=\phi_{O}-$ $\phi_{R} ;$ and $k=2 \pi / \lambda$ ( $\lambda$ being the optical wavelength). The fringe spacing $\Lambda$ is then given by the well-known relation

$$
\Lambda=\lambda /(2 \cos \theta)
$$




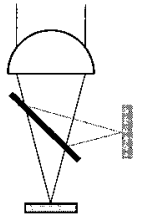

Michelson

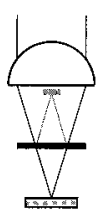

Mirau

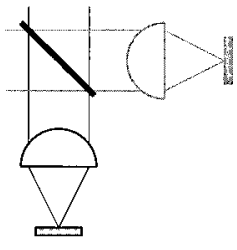

Linnik
Fig. 1. Geometries of interference microscopes where object and reference surfaces are illuminated with converging beams: Michelson, Mirau, and Linnik. The drawback in the Michelson and the Mirau configurations is that the beam splitter can introduce strong aberrations in wide-aperture systems, especially the spherical aberration. Because reference and object beams share a common path over most of their length, these layouts are less sensitive to vibrations than is the Linnik configuration. In addition, only one lens is required, although its working distance must be long enough to accommodate the beam splitter. The Linnik configuration requires two identical objectives, and, because of the long beam paths involved, it must be built massively to avoid vibration problems. Nevertheless, the great advantage of this configuration is that objectives with high numerical apertures can be used. We chose this configuration in our experiments described in Section 3.

The fringe spacing $\Lambda$ does not depend on the optical path difference $z$. It is reduced to $\lambda / 2$ in the case of normal incidence $(\theta=0)$, and it increases toward infinity when $\theta$ approaches $\pi / 2$.

In interference microscopes (such as Michelson, Mirau, or Linnik microscopes shown in Fig. 1), the object and the reference surfaces are illuminated with a converging beam. Therefore no single angle $\theta$ can characterize the angle of incidence. The incidence $\theta$ actually varies from 0 to $\theta_{\max }$, where $\theta_{\max }$ is related to the numerical aperture of the objective lenses by $\mathrm{NA}=\sin \theta_{\max }$ (in air). To calculate the actual interferogram, the contribution of each ray of the converging cone of rays at each angle of incidence must be appropriately weighted and summed over the entire angular aperture of the microscope objective. Assuming that the reflectivity of the mirrors does not vary with the incidence angle $\theta$ (which is a good approximation with metallic mirrors in the visible region of the spectrum), the interference signal intensity $I(z)$ then takes the more complicated form $^{3,10}$

$$
I(z)=A_{O}^{2}+A_{R}^{2}+2 A_{O} A_{R} F_{\mathrm{NA}}(z),
$$

with

$$
\begin{aligned}
F_{\mathrm{NA}}(z)= & \frac{2}{\sin ^{2} \theta_{\max }} \int_{0}^{\theta_{\max }} \cos (2 k z \cos \theta+\phi) \\
& \times \cos \theta \sin \theta \mathrm{d} \theta .
\end{aligned}
$$

It is worth noting that a rigorous analysis with the Richards and Wolf vector theory of light ${ }^{11,12}$ yields the same expression as in Eq. (4). The integral in Eq. (4) can be calculated analytically, leading to

$$
\begin{aligned}
& F_{\mathrm{NA}}(z)=\frac{2}{\sin ^{2} \theta_{\max }} \\
& \times\left[\begin{array}{c}
\frac{\cos (2 k z+\phi)-\cos \left(2 k z \cos \theta_{\max }+\phi\right)}{4 k^{2} z^{2}} \\
+\frac{\sin (2 k z+\phi)-\cos \theta_{\max } \sin \left(2 k z \cos \theta_{\max }+\phi\right)}{2 k z}
\end{array}\right] .
\end{aligned}
$$

In the case of low numerical aperture $\left(\theta_{\max } \approx 0\right)$, the function $F_{\mathrm{NA}}(z)$ is reduced to the usual expression

$$
F_{\mathrm{NA}}(z)=\cos (2 k z+\phi),
$$

which is in agreement with Eq. (1) in the case of normal incidence $(\theta=0)$. The function $F_{\mathrm{NA}}(z)$ given in Eq. (5) is plotted in Fig. 2 for different numericalaperture values. $F_{\mathrm{NA}}(z)$ exhibits oscillations whose amplitude decreases with $|z|$ with lobes. The envelope of the oscillations becomes increasingly narrow as the numerical aperture increases. A simpler and more explicit expression for $F_{\mathrm{NA}}(z)$ can be given under the paraxial assumption $\left(\cos \theta_{\max } \approx 1\right)$,

$$
F_{\mathrm{NA}}(z) \approx \frac{2}{\sin ^{2} \theta_{\max }} \int_{0}^{\theta_{\max }} \cos (2 k z \cos \theta+\phi) \sin \theta \mathrm{d} \theta,
$$

which can be solved analytically as

$$
\begin{aligned}
F_{\mathrm{NA}}(z) \approx & \frac{2}{1+\cos \theta_{\max }} \frac{\sin \left[k z\left(1-\cos \theta_{\max }\right)\right]}{k z\left(1-\cos \theta_{\max }\right)} \\
& \times \cos \left[k z\left(1+\cos \theta_{\max }\right)+\phi\right] .
\end{aligned}
$$

Thus $F_{\mathrm{NA}}(z)$ can be approximated as

$$
F_{\mathrm{NA}}(z) \approx \gamma_{\mathrm{NA}}(z) \cos \left[2 \alpha_{\mathrm{NA}} k z+\phi\right],
$$

where

$$
\begin{aligned}
\gamma_{\mathrm{NA}}(z) \propto \frac{\sin \left[k z\left(1-\cos \theta_{\max }\right)\right]}{k z\left(1-\cos \theta_{\max }\right)}, \\
\alpha_{\mathrm{NA}}=\left(1+\cos \theta_{\max }\right) / 2 .
\end{aligned}
$$

One can see from relations (9)-(11) that $F_{\mathrm{NA}}(z)$ has an approximately sinusoidal variation of period modified by an aperture factor $\alpha_{\mathrm{NA}}$ multiplied by an envelope $\gamma_{\mathrm{NA}}(z)$ that has a maximum at $z=0$ and falls off when $|z|$ increases like a $(\sin x / x)$ function. The fringe spacing $\Lambda$ is thus approximately given by

$$
\Lambda \approx \frac{\lambda}{2 \alpha_{\mathrm{NA}}}=\frac{\lambda}{2} \frac{2}{\left(1+\cos \theta_{\max }\right)}=\frac{\lambda}{2} \frac{2}{[1+\cos (\arcsin \mathrm{NA})]},
$$



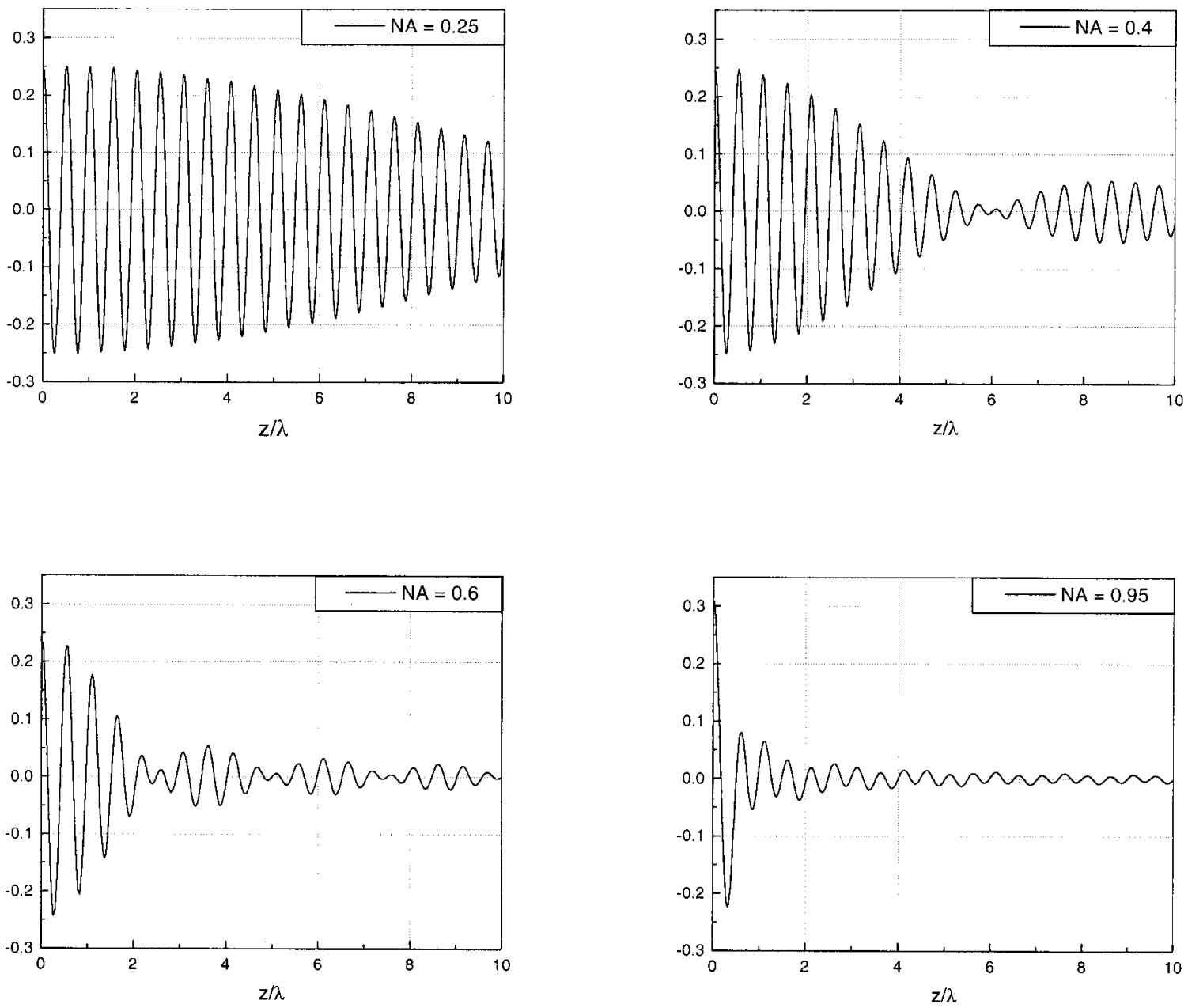

Fig. 2. Plots of the function $F_{\mathrm{NA}}(z)$ for different numerical-aperture values [from Eq. (5)]. The displacement of the reference mirror (along $z$ ) is normalized by the optical wavelength.

and the FWHM of the envelope $\gamma_{\mathrm{NA}}(z)$ is given by

$$
\mathrm{FWHM}_{\gamma_{\mathrm{NA}}} \approx \frac{0.6 \lambda}{\left(1-\cos \theta_{\max }\right)}=\frac{0.6 \lambda}{[1-\cos (\arcsin \mathrm{NA})]}
$$

From relation (12) one can see that the fringe spacing $\Lambda$ increases nonlinearly with the numerical aperture, varying from $\Lambda=\lambda / 2$ for normal incidence $(\mathrm{NA}=0)$ to $\Lambda=\lambda$ for $\mathrm{NA}=1$. The true theoretical fringe spacing $\Lambda$ was obtained graphically from plots of the exact expression of $F_{\mathrm{NA}}(z)$ [Eq. (5)] near $z=0$. The discard between the exact and the approximate fringe spacing increases with the numerical aperture, the exact fringe spacing actually varying from $\Lambda=$ $\lambda / 2$ (just like the approximate) for $\mathrm{NA}=0$ to $\Lambda \approx 0.7$ $\lambda$ [rather than $\lambda$ given by the approximate relation (12)] for NA $=1$. The fringe spacing variation with numerical aperture is shown in Fig. 3(a) with the exact and the approximate calculations.

The fringe envelope also varies with the numerical aperture. Relation (13) shows that the envelope gets narrower as the numerical aperture increases.
We can find the true envelope of the fringes, i.e., the envelope of the function $F_{\mathrm{NA}}(z)$ in Eq. (5) numerically by carrying out a discrete Fourier transform of a set of values of $F_{\mathrm{NA}}(z)$ and eliminating the negative spatial frequencies. Then the packet of positive spatialfrequency components is centered, and an inverse discrete Fourier transform is calculated. The approximate and the exact FWHM's of the fringe envelope are close except for extremely high numerical apertures as shown in Fig. 3(b).

The variation of the fringe spacing with the numerical aperture should be taken into account for accurate phase measurements. For example, in applications in which the phase is used for height measurements, taking $\Lambda=\lambda / 2$ as with interferometers that use plane waves in normal incidence yields underestimation of heights. To obtain accurate height measurement, the optical wavelength should be multiplied by an appropriate corrective factor $\beta$. Equation (11) provides an approximate expression of $\beta$ as

$$
\beta \approx \frac{1}{\alpha_{\mathrm{NA}}}=\frac{2}{[1+\cos (\arcsin \mathrm{NA})]}
$$




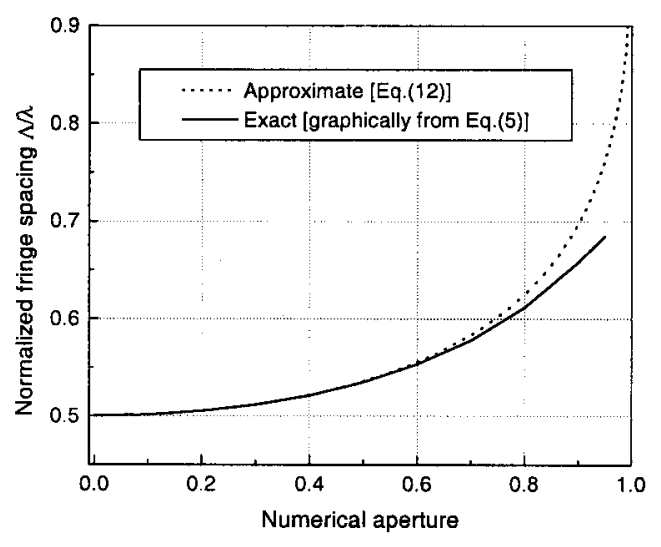

(a)

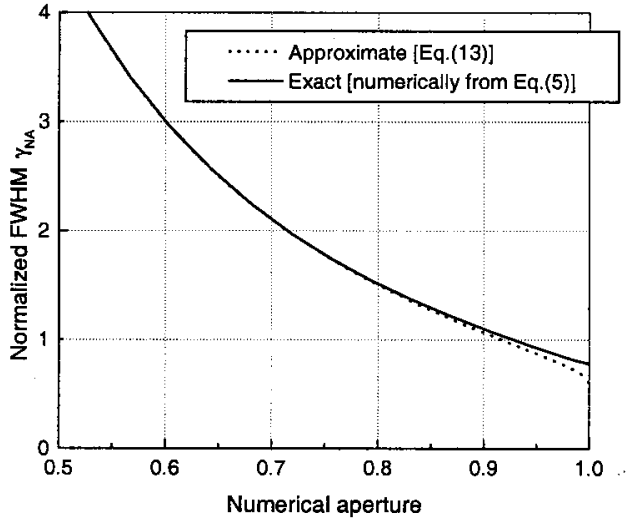

(b)

Fig. 3. (a) Theoretical fringe spacing variation with numerical aperture from exact and approximate theories (normalized by the optical wavelength). (b) Theoretical FWHM of the fringe envelope from exact and approximate theories (normalized by the optical wavelength).

Let us develop $1 / \alpha_{\mathrm{NA}}$ in power series as

$$
\frac{1}{\alpha_{\mathrm{NA}}}=1+\frac{1}{4}(\mathrm{NA})^{2}+\frac{1}{8}(\mathrm{NA})^{4}+\frac{5}{64}(\mathrm{NA})^{6}+O(\mathrm{NA})^{7} .
$$

More precise values of the corrective factor $\beta$ can be obtained numerically from Eq. (5). Then one can express $\beta$ as a function of numerical aperture, using the development in Eq. (15) to fit the calculated points by adjusting some coefficients,

$$
\beta=1+0.25(\mathrm{NA})^{2}+0.1(\mathrm{NA})^{4}+0.086(\mathrm{NA})^{6} .
$$

With the development given in Eq. (16) the fit is excellent $\left(\chi^{2}=8 \cdot 10^{-7}\right)$. Values of the corrective factor $\beta$ are given in Table 1 for different numerical apertures with relations (14) and (16). Significant differences appear between approximate and exact values of $\beta$ for high numerical apertures.

As discussed above, in addition to fringe spacing variation with numerical aperture, the fringe contrast decreases with $|z|$ approximately as the $\gamma_{\mathrm{NA}}(z)$ function given in Relation (10), which means that heights can be measured only within a limited range $\left[\sim \mathrm{FWHM}\right.$ of $\left.\gamma_{\mathrm{NA}}(z)\right]$. These results are reported in Table 1. Discards between approximate and exact calculation are small even for high numerical apertures.

Experiments were carried out to check the validity of these theoretical investigations.

\section{Experiments}

Experiments were carried out with a homemade Linnik-type interference microscope, which permits use of high numerical apertures as discussed above (see Fig. 1). Our experimental arrangement is schematically represented in Fig. 4. A He-Ne laser at $633 \mathrm{~nm}$ was used as the optical source. Its beam was spatially filtered and expanded to fill the whole back aperture of the objective lenses. Metallic mirrors were used as object and reference surfaces. The interference signal intensity was recorded with a silicon photodiode while the mirror in the object arm of the interferometer was moved axially (along $z$ ). Dry

\begin{tabular}{|c|c|c|c|c|}
\hline NA in Air & $\begin{array}{c}\text { Corrective Factor } \beta \\
\text { from Relation (14) } \\
\text { (approximate) }\end{array}$ & $\begin{array}{l}\text { Corrective Factor } \beta \\
\text { from Eq. (16) }\end{array}$ & $\begin{array}{l}\text { Normalized FWHM of } \\
\text { the Fringe Envelope } \\
\text { from Relation (13) } \\
\text { (approximate) }\end{array}$ & $\begin{array}{c}\text { Normalized FWHM of } \\
\text { the Fringe Envelope } \\
\text { from Eq. (5) }\end{array}$ \\
\hline 0 & 1 & 1 & $\infty$ & $\infty$ \\
\hline 0.1 & 1.0025 & 1.0025 & 120 & 120 \\
\hline 0.2 & 1.0102 & 1.0102 & 29.7 & 29.7 \\
\hline 0.3 & 1.0236 & 1.0234 & 13.0 & 13.0 \\
\hline 0.4 & 1.0436 & 1.0429 & 7.2 & 7.2 \\
\hline 0.5 & 1.0718 & 1.0699 & 4.5 & 4.5 \\
\hline 0.6 & 1.1111 & 1.1064 & 3.0 & 3.0 \\
\hline 0.7 & 1.1668 & 1.1556 & 2.1 & 2.1 \\
\hline 0.8 & 1.2500 & 1.2232 & 1.50 & 1.51 \\
\hline 0.9 & 1.3929 & 1.3160 & 1.06 & 1.09 \\
\hline 0.95 & 1.5241 & 1.3688 & 0.87 & 0.93 \\
\hline
\end{tabular}

Table 1. Corrective Factor $\boldsymbol{\beta}^{a}$ and Measurable Range of Height ${ }^{b}$

${ }^{a}$ For multiplication with optical wavelength for different numerical apertures.

${ }^{b}$ Normalized by optical wavelength. 


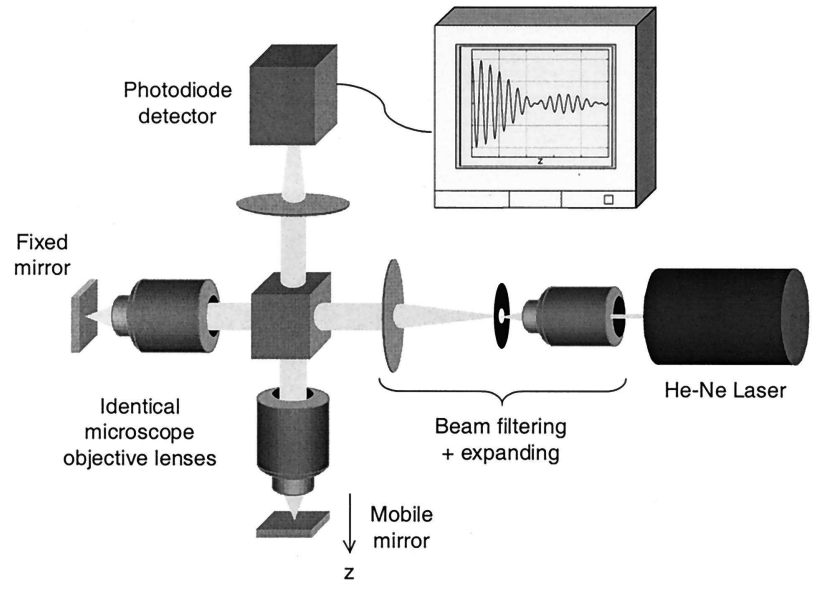

Fig. 4. Experimental arrangement: a homemade Linnik-type interference microscope illuminated with a $\mathrm{He}-\mathrm{Ne}$ laser whose beam has been spatially filtered and expanded to fill the whole back aperture of the objective lenses.

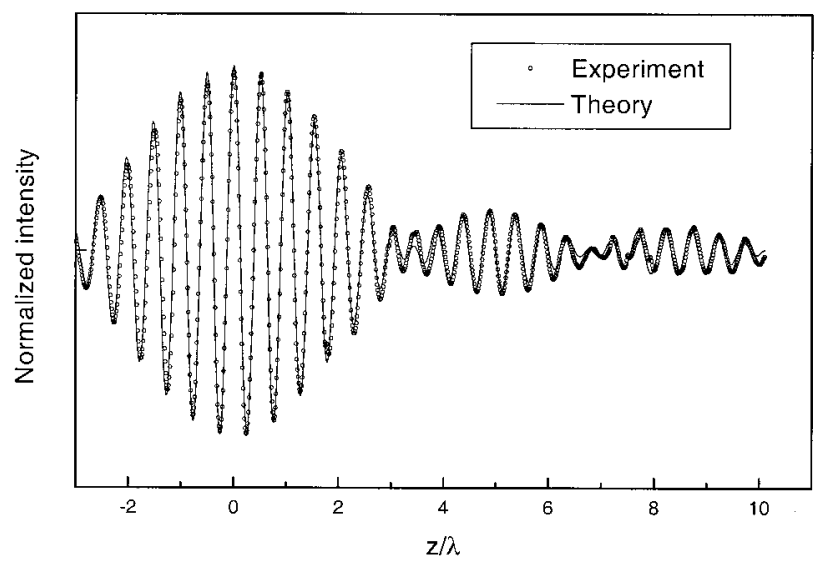

Fig. 5. Experimental and theoretical interferograms obtained with our Linnik-type interference microscope with 0.5-NA objective lenses. In the theoretical curve no parameter was adjusted to fit the experimental data.

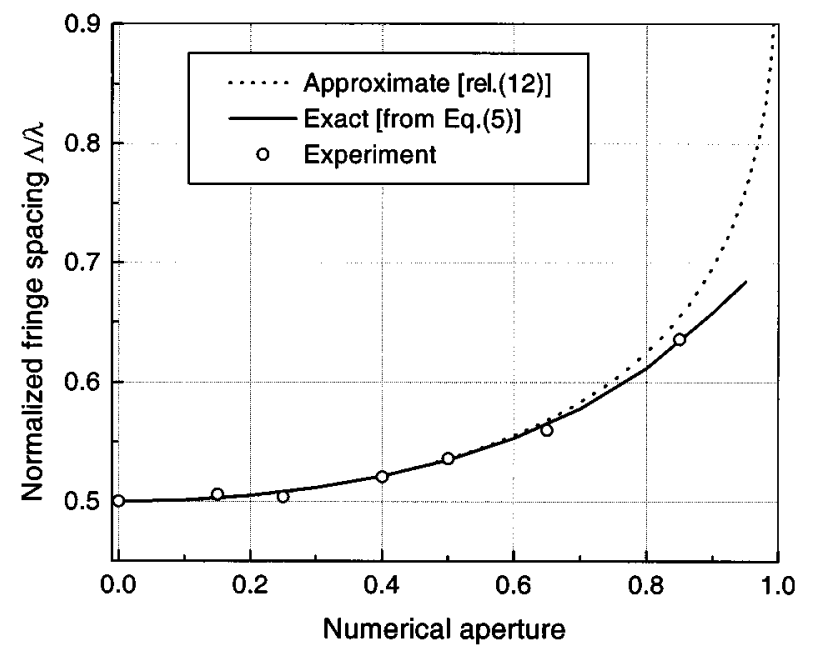

Fig. 6. Theoretical and experimental fringe spacing versus numerical aperture, normalized by the optical wavelength. No parameter was adjusted to fit the experimental data.

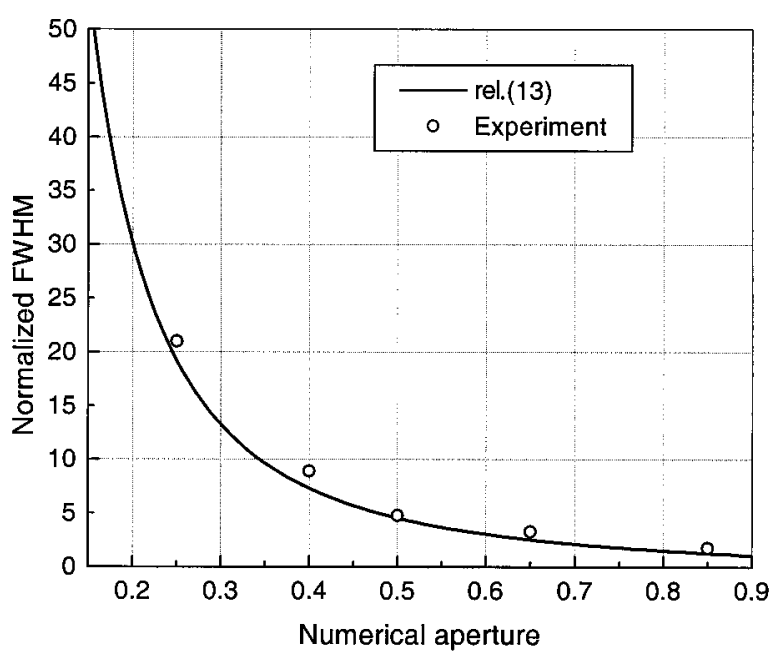

Fig. 7. Theoretical and experimental FWHM of the fringe envelope versus numerical aperture, normalized by the optical wavelength. No parameter was adjusted to fit the experimental data.

objective lenses with different numerical apertures were used.

An example of an interferogram obtained with 0.5-NA objectives is shown in Fig. 5. The agreement with theory is excellent, although no parameter in the theoretical curve was adjusted to fit the experimental data.

From all the experimental interferograms, we measured the fringe spacing near $z=0$. The results, presented in Fig. 6, are in good agreement with the exact theory, whereas discards are observed from the approximate theory at high numerical apertures, as expected. The envelope of the experimental intensity responses were calculated just as in the theoretical study. A discrete Fourier transform was carried out, and the negative spatial frequencies were eliminated. Then the packet of positive spatialfrequency components was centered, and an inverse discrete Fourier transform was calculated. The FWHM's of the experimental envelopes of the fringes are presented in Fig. 7. The agreement with theory is good.

\section{Conclusion}

We have presented a theoretical study of the interferogram produced by wide-aperture interferometers. We have shown that the fringe spacing increases nonlinearly with the numerical aperture. Phase measurements in wide-aperture interferometers therefore require calibration. The wavelength should be multiplied by a corrective factor $\beta$. An approximate expression of $\beta$ as a function of the numerical aperture was given, and a more precise expression was proposed with a power-series expansion. Because of the extinction of the fringe contrast with the optical path difference, the phase can be measured over a limited range, which is reduced to a few fringes in the case of high numerical apertures. However, this unavoidable phenomenon does not influence measurement accuracy once fringe spacing 
correction is applied. Experimental measurements have shown good agreement with theory.

\section{References}

1. F. Laeri and T. C. Strand, "Angström resolution optical profilometry for microscopic objects," Appl. Opt. 26, 2245-2249 (1987).

2. K. Creath, "Phase-measurements interferometry techniques," in Progress in Optics, E. Wolf, ed. (North Holland, Amsterdam, 1988), Vol. XXVI, pp. 349-393.

3. G. S. Kino and S. C. Chim, "Mirau correlation microscope," Appl. Opt. 29, 3775-3783 (1990).

4. J. F. Biegen, "Calibration requirements for Mirau and Linnik microscope interferometers," Appl. Opt. 28, 1972-1974 (1989).

5. F. R. Tolmon and J. G. Wood, "Fringe spacing in interference microscopes," J. Sci. Instrum. 33, 236-238 (1956).

6. C. F. Bruce and B. S. Thornton, "Obliquity effects in interference microscopes,” J. Sci. Instrum. 34, 203-204 (1956).
7. J. W. Gates, "Correspondence: fringe spacing in interference microscopes," J. Sci. Instrum. 33, 507 (1956).

8. T. Wilson, R. Juskaitis, N. P. Rea, and D. K. Hamilton, "Fibre optic interference and confocal microscopy," Opt. Commun. 110, 1-6 (1994).

9. D. K. Hamilton and J. R. Sheppard, "Interferometric measurements of the complex amplitude of the defocus signal $V(z)$ in the confocal scanning optical microscope," J. Appl. Phys. 60, 2708-2712 (1986).

10. C. J. R. Sheppard and T. Wilson, "Effects of high angles of convergence on $V(z)$ in the scanning acoustic microscope," Appl. Phys. Lett. 38, 858-859 (1981).

11. B. Richards and E. Wolf, "Electromagnetic diffraction in optical systems: II. Structure of the image field in an aplanetic system," Proc. Soc. London Ser. A 253, 358-379 (1959).

12. F. C. Chang and G. S. Kino, "325-nm interference microscope," Appl. Opt. 37, 3471-3479 (1998). 